\title{
¿“Gongoresk"? Borges, su primera recepción alemana y la tentación de su lectura manierista
}

Mucho ya se ha escrito sobre la difusión mundial ${ }^{1}$ de la obra de Jorge Luis Borges que, pese a haberse iniciado ya en los años 1920 con la elogiosa reseña de sus Inquisiciones por V. Larbaud ${ }^{2}$, arrancó realmente con la publicación de sus textos en la revista Sur y, a partir de allí, con la proyección internacional de su obra, especialmente en Francia ${ }^{3}$. La primera recepción de la obra de Jorge Luis Borges en la Alemania de los años 1950-1960, que depende fuertemente de la francesa que la precede, representa un buen caso de análisis de las interacciones entre los distintos actores del proceso editorial cuyos papeles respectivos se estudian en los trabajos aquí reunidos. Ya están documentados en muchos de sus aspectos los detalles de esta "recepción" - palabra que conviene usar con toda cautela-: ahí están los trabajos de Siebenmann, Broyles-González, Aizenberg y, más recientes, los de Gerling y Pérez Blázquez ${ }^{4}$. Un lugar destacado entre ellos merecería Gutiérrez Girardot, pionero por haber sido a la vez actor e historiador de este proceso, y que propuso una retrospectiva de su visión de la recepción alemana de Borges en 1976 - se le evocará más adelante $-{ }^{5}$. Este estu-

1 Sobre la problemática de la literatura mundial y de la mundialización de la literatura, en la que le incumbe a Borges un lugar nada desdeñable - como actor así como síntoma-, se puede remitir a los planteamientos de Marcelo Topuzian, que discuten de modo productivo las posturas de Moretti y Casanova (Topuzian 2014).

2 Véase Larbaud (1925, 1976 y 2011).

3 Véase en especial Molloy (1972: 194-247), así como Louis (2007) y Klengel (2013).

4 Siebenmann (1966, 1992, 1996); Broyles-González (1981); Aizenberg (1990); Gerling (2015); Pérez Blázquez (2018).

5 Ya le dedicaba todo un ensayo en 1959: Gutiérrez Girardot (1959), y luego publicó reseñas críticas: Gutiérrez Girardot (1962). Para su retrospectiva, véase Gutiérrez Girardot (1976), que retoma Gutiérrez Girardot (1964). En 1964, además, escribió un epílogo para la traducción de unos cuentos de Borges en la prestigiosa serie "Insel-Bücherei” (Borges 1964).

Note: Agradezco a Annick Louis y Gustavo Guerrero su atenta lectura y su ayuda para la descripción de las traducciones de R. Caillois.

Roland Béhar, ENS-PSL

Ә Open Access. (c) 2021 Roland Béhar, published by De Gruyter. (cc))BY-NC-ND This work is licensed under a Creative Commons Attribution-NonCommercial-NoDerivatives 4.0 International License.

https://doi.org/10.1515/9783110713015-011 
dio se limitará, por tanto, a un breve recuento de las primeras etapas de la historia de los textos de Borges en Alemania - RFA, que no RDA, según se verá-, para centrarse luego en los discursos que se desarrollaron al mismo tiempo acerca de este proceso. Se mostrará cómo se dio una evolución paralela y una - relativa, por supuesto - rivalidad entre ámbitos editoriales y ámbitos universitarios, para mostrar cómo la imagen de Borges se halló suspendida entre los polos opuestos de la latinoamericanidad y de la universalidad.

En 1951, la editorial Gallimard publicó Ficciones, en la colección La Croix $d u$ Sud, fundada y dirigida por Roger Caillois ${ }^{6}$. Algunos de los textos de este primer libro de Borges en lengua francesa, traducido por Néstor Ibarra y Paul Verdevoye, ya habían sido publicados en revistas, en Lettres françaises ${ }^{7}$ y en Confluences $^{8}$. Dos años después, en 1953, Gallimard publicó Labyrinthes, pero, curiosamente, no en la misma colección La Croix du Sud ${ }^{9}$. En este libro, Roger Caillois tradujo una selección de cuentos de El Aleph (cuya primera edición como libro se remontaba a 1949): "L'immortel”, "Histoire du guerrier et de la captive”, “L'écriture du dieu” y “La quête d'Averroës”. Como lo muestra Annick Louis, Labyrinthes "se volvió el libro esencial al conocimiento de Borges, proponiendo al laberinto como un prisma interpretativo a través del cual será percibida y comprendida su obra" ${ }^{\text {}}$. Esto se debe entre otras cosas a que Caillois escribió un prefacio para Labyrinthes que volvería a publicar en 1967, ampliándolo, a guisa de prefacio de la versión francesa completa de El Aleph. Después de Fictions, que respetó la forma inicialmente establecida por Borges a su colección de relatos, el Borges de Caillois fue un Borges modificado y transformado, no en los mismos textos, pero sí en la significación de conjunto que les otorga

\footnotetext{
6 Borges (1951).

7 Sobre la política editorial de esta revista, véase Louis (2020b).

8 Borges (1944, 1946). Es de notar, además, que Borges ya había sido traducido en 1939, con la publicación en la revista Mesures de la traducción por Néstor Ibarra de El acercamiento a Almotásim (publicado por Borges en la Historia de la eternidad, de 1936), bajo el título "L'Approche du caché". Sobre Ibarra traductor, véase Batalla (2010). Otro caso de temprana difusión, pero que nos interesa menos aquí en relación con la recepción alemana, es la italiana, que se centra en la poesía vanguardista del joven Borges, traducida entre 1927 y 1937 (Gavag$\operatorname{nin} 2018)$.

9 Borges (1953a).

10 Louis (2020a) - agradezco a la autora la lectura de su estimulante ensayo antes de su publicación-. Sobre Caillois y Borges en general, véase Louis (2002, 2007 y 2013).
} 
su reorganización -apropiación que no carece de sal, si se recuerda la polémica entre Borges y Caillois de inicios de los años 1940, en torno a la definición de la novela policial-.

Este Borges resulta ser bastante "barroco" o mejor, según se verá, "manierista.” Basta echarle un ojo a la portada, para la cual -el detalle tiene su interésla editorial Gallimard utiliza, si no por indicación de Caillois, cuando menos con su beneplácito ${ }^{11}$, una imagen de un laberinto muy precisa (Figure 1).

¿Cómo llegó a materializarse esta idea de cubierta? La imagen, no cabe duda, le venía al dedillo al proyecto de libro tal como Caillois lo diseñó: es una foto del famoso techo de la llamada Sala del Laberinto del palacio ducal de Mantua, con el motto "Forse che sì, forse che no" ${ }^{2}$. Ahora bien, este mismo motto de Vincenzio Gonzaga, duque de Mantua, ya había fascinado a Gabriele D’Annunzio, quien lo utilizó para el título de Forse che sì, forse che no (1910). En esta novela ambientada, con abundantes descripciones, en la Mantua renacentista, D’Annunzio había celebrado el arte de la aviación y, con ello, el vuelo icárico, respondiendo así al incipiente futurismo de Marino. Significativamente, la cubierta de la novela reproducía, bajo forma de grabado, el dibujo del laberinto del techo del palacio (Figure 2), y el encabezado del primer capítulo retomaba luego el mismo motivo (Figure 3):

¿Tenía conocimiento Caillois de la novela de D’Annunzio, cuya traducción francesa fue publicada por Calmann-Levy el mismo año de su publicación original,

11 Una consulta de los archivos de la casa editorial, a través de la amable ayuda de Éric Legendre, no ha podido arrojar nueva luz sobre este detalle de la historia de la publicación del libro.

12 En su estudio exclusivamente dedicado a las cubiertas de las ediciones castellanas y traducidas de Ficciones y de El Aleph, Brigitte Adriaensen y Lies Wijnterp no toman en cuenta este origen de la imagen: "La cubierta muestra por primera vez la figura de un laberinto en un libro de Borges, un motivo que se utilizará con mucha frecuencia después. El laberinto está en gris y blanco, con letras rojas. De nuevo, el ilustrador no está indicado ni es conocido por Gallimard" (Adriaensen/Wijnterp 2013: 14). Esto resulta sorprendente, cuando ya en la época de su publicación la alusión de la portada no había pasado desapercibida. Un reseñista entusiasmado escribió en 1954: "Car, qu'est-ce que le cosmopolitisme, sinon une forme du détachement? Borges est en effet l'un des derniers 'détachés' de ce monde, avec Jünger, par exemple. Forse che sì, forse che no, telle pourrait être sa devise” (Anón. 1954: 163). Y, después de comparar - para destacar mejor su singularidad- a Borges con otros "détachés," como Romain Rolland, Pierre Drieu La Rochelle y André Gide, concluye: "Borges avance sur le fil tendu de l'ambiguïté : non, il n'est pas un 'écrivain éternel,' un tenant de l'art pour l'art, etc.: il vient après cela. Borges est-il l'écrivain de demain? Non, de jamais. Qu'importe qu'on le découvre dans cinq siècles . . . " (Anón. 1954: 164). 


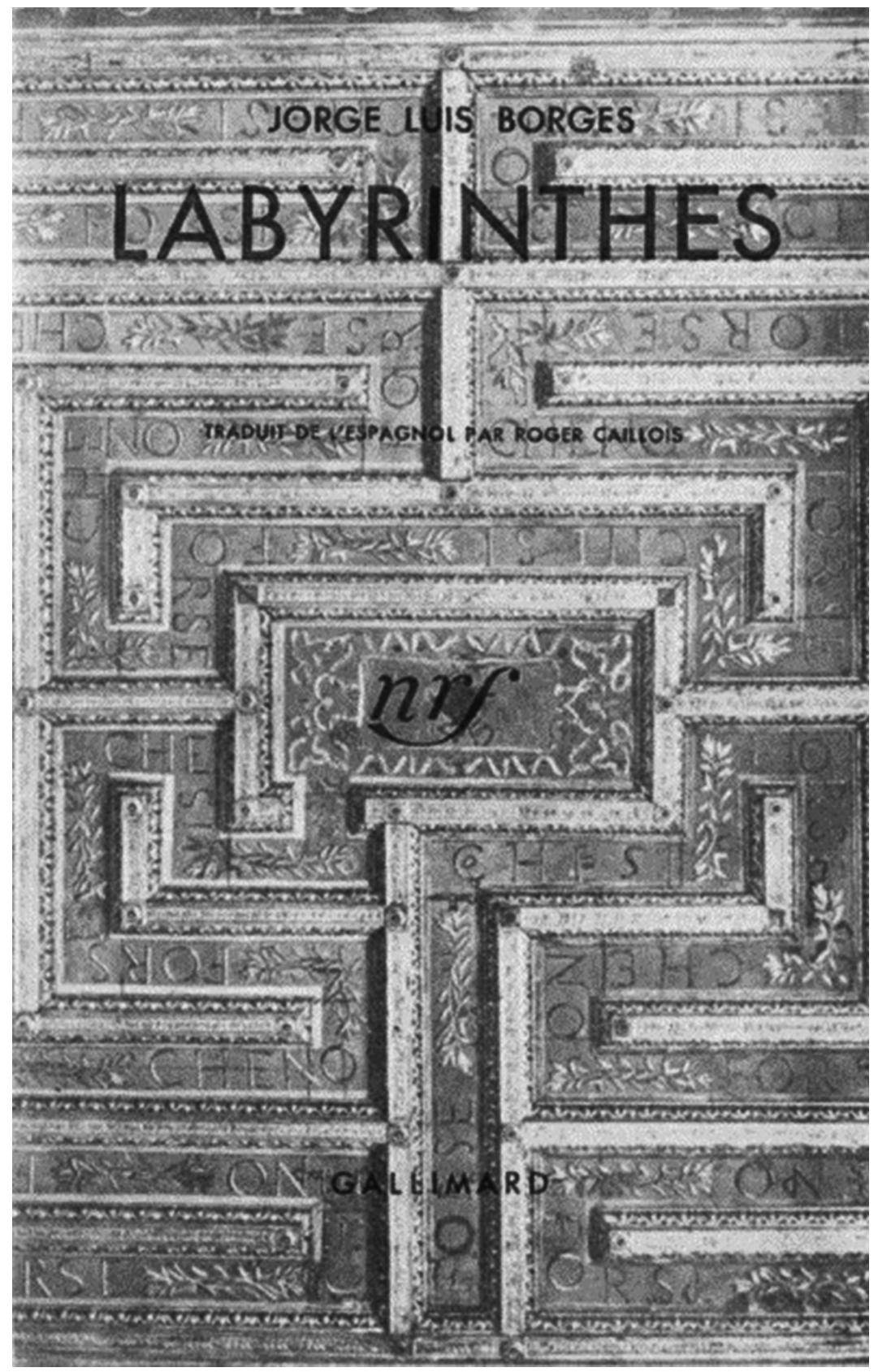

Figure 1: Portada de Jorge Luis Borges, Labyrinthes. París: Gallimard, 1953. 


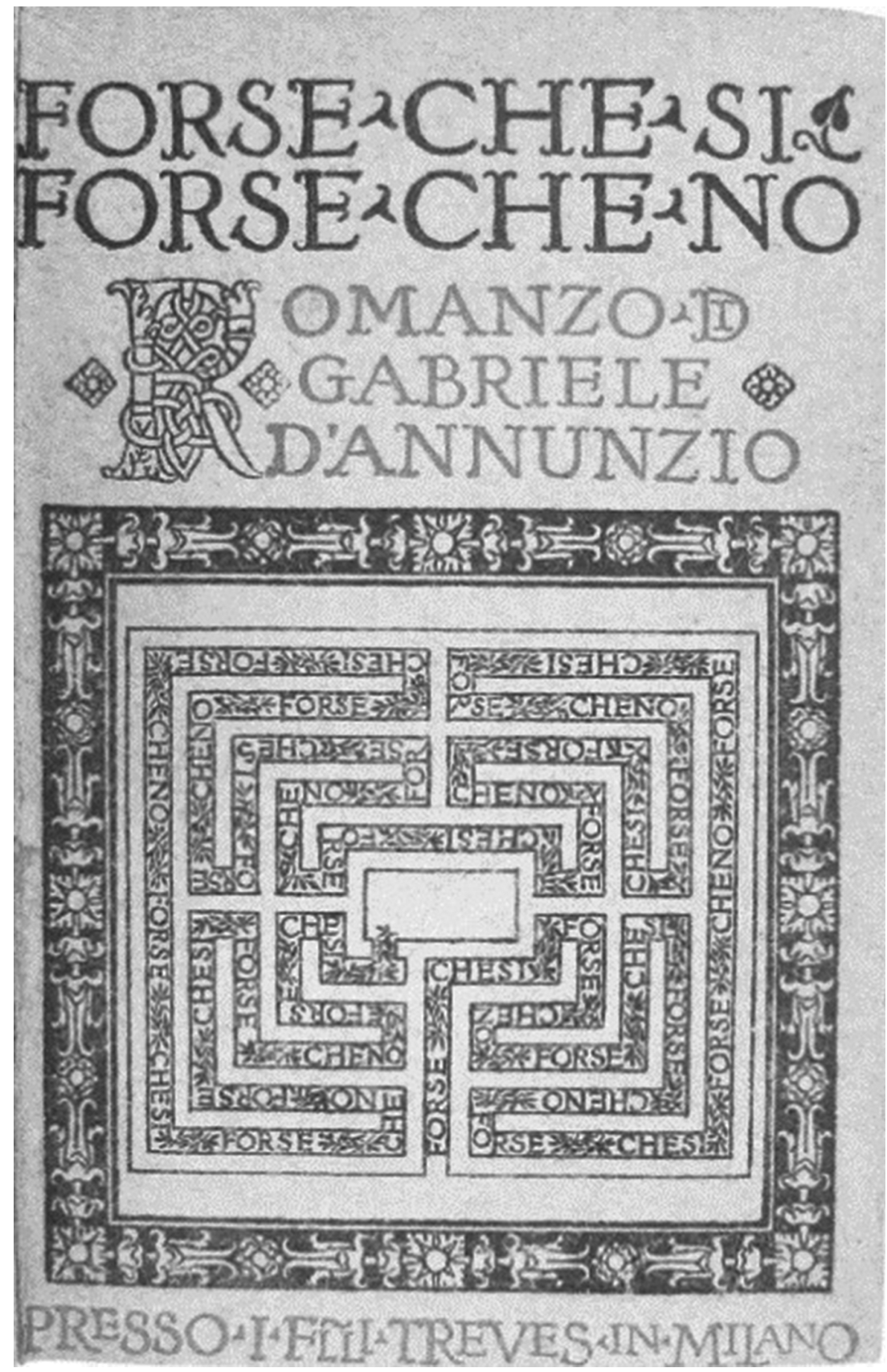

Figure 2: Portada de Gabriele D’Annunzio, Forse che sì, forse che no. Milán: Fratelli Treves, 1910. 


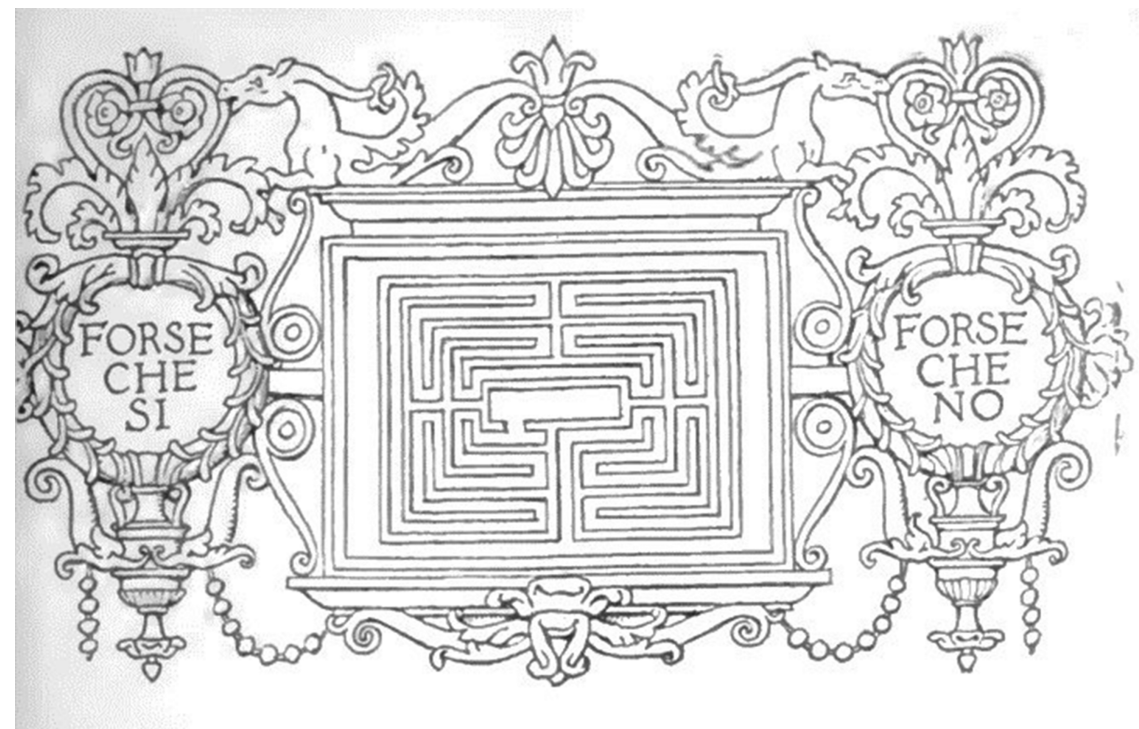

Figure 3: Gabriele D’Annunzio, Forse che sì, forse che no. Encabezado del capítulo I, p. 1.

el día del solsticio de 1910, y que suscitó inmediatamente muchos admiradores ${ }^{13}$ ? Resulta difícil determinarlo, pero igual de arduo sería negar su alta probabilidad, considerando el prestigio del que gozaba aún D’Annunzio en los años 1920 y $1930^{14}$. Otra vía por la que Caillois podría haber tenido conocimiento del laberinto mantuano -dando por descontada su ingente cultura personal-sería su amigo el historiador del arte André Chastel, grandísimo experto del Renacimiento italiano,

13 La novela fue alabada por Robert de Montesquiou, quien le dedicó una conferencia, y por Marcel Proust, quien la calificó, en su correspondencia con el mismo Montesquiou, como “merveilleux roman” (Proust 1970-1993: vol. X, 222-223, y vol. XVI, 150). Otro lector, algo más crítico, fue Israel Zangwill, quien evocó a su vez, en sus Italian fantasies (1910), la Mantua celebrada por D’Annunzio, recordando, para denunciarlo, que el italiano había usado la imagen del techo del palacio para su cubierta (se cita por la traducción francesa del libro, que Caillois bien pudo leer): "Et, se répétant en alternances régulières avec le labyrinthe, la devise que d'Annunzio a empruntée pour un de ses romans: 'Forse che si, forse che no . . . ' qu'il a reproduite en titre sur la couverture” (Zangwill 1924: 8). El título de la traducción francesa (“Avec une pierre dans le jardin de d'Annunzio ... ") es menos explícito que el título original (“Lachrymæ rerum at Mantua: With a Denunciation of d'Annunzio", en Zangwill 1910).

14 Véase por caso a Gaston Bachelard (1884-1962), amigo a quien Caillois conocía desde los años 1930: recurre a D’Annunzio en El agua y los sueños, de 1941 (Bachelard 2011), cuando da un ejemplo sacado de Forse che sì, forse che no. Sobre Bachelard lector de D’Annunzio, véase Tuzet (1984). 
con quien había trabado una estrecha amistad desde los primeros tiempos de sus estudios universitarios. ¿Quiso Caillois aludir de alguna manera al libro de D’Annunzio? Difícil pregunta, pero que apunta en todo caso a una perspectiva interpretativa en continuidad con los gustos modernistas de D’Annunzio.

Lo que sí se sabe es cómo Caillois interpretó el laberinto según Borges. La contraportada de Labyrinthes justificó su elección:

La rigueur du contenu, une logique implacable et déconcertante ne le cèdent en rien à la perfection de la forme et à la richesse de l'invention. Un conte de Borges est une mécanique de précision où la moindre pièce joue son rôle.

Y Caillois, a renglón seguido, describe la tarea que le correspondió como traductor, mostrando similitudes entre Borges y los escritores españoles del Siglo de Oro:

La tâche du traducteur n'était pas moins complexe et nouvelle. Ainsi tel récit est écrit dans un style qui imite l'espagnol baroque du XVII ${ }^{\mathrm{e}}$ siècle, en particulier la langue de Quevedo. En outre, il est donné comme une traduction de l'anglais. Enfin, il est nécessaire qu'il présente d'assez nombreux latinismes de vocabulaire et de syntaxe. La traduction joue ainsi simultanément sur quatre langues : latin, anglais (en principe), espagnol et français. Elle doit rendre des tours très particuliers à la prose de l'auteur. D'où, parfois, des préciosités d'expression, toujours calculées, mais qui peuvent néanmoins surprendre le lecteur pressé. C'est d'ailleurs en partie le goût de résoudre ces rares difficultés qui a déterminé Roger Caillois à tenter l'entreprise.

Todo libro pertenece a sus lectores: el lector/traductor Caillois quiso en cierta medida apropiarse el libro de Borges, imponiéndole su impronta y haciendo alarde de virtuosismo literario, para lo cual resaltó el "barroquismo" de los textos de Borges - por decirlo con el término del propio Borges-. Este iba a ser el punto de partida de una oleada de lecturas que podrían llamarse barroquizantes, en Francia y más allá, que se apoyarían en este y en otros elementos, como se mostrará para el caso alemán. El mérito de Caillois en esta caracterización del autor argentino es, sin embargo, muy relativo, porque esta asociación de Borges con la imagen del laberinto es algo que ya se vislumbra en uno de los primeros textos dedicados a Borges por Pierre Drieu la Rochelle - quien escribiera, en 1933, la frase célebre "Borges vaut le voyage"-: "Villes du monde. Solitude de Buenos-Ayres" (1934) $)^{15}$. Y cabe adelantar que la motivación estética que propone Caillois para la lectura de

15 Véase Pierre Drieu la Rochelle (1934: 6): “Dès mon arrivée, j’ai rencontré un poète argentin qui a tout de suite voulu me donner sa ville dans tout son excès, dans toute sa grandeur, dans tout son caractère. Georges-Louis Borges m'a fait prendre le métro. Nous avons mis le nez dehors à une station quelconque, vers minuit, et, sous une lune énorme et diluée, nous avons commencé d'errer dans cet immense labyrinthe rectiligne. Nous marchions comme sur une carte, sur une épure, sans repères humains. Nous étions en plein dans l'abstraction. Des ave- 
Borges a inicios de los años 1950 no se puede, sin embargo, desligar del todo de una percepción política del texto de Borges, sobre la que se volverá más adelante.

Pero no es sino en 1961 - año en que Borges se ve galardonado, junto con Samuel Beckett, con la primera edición del "Prix International de Littérature"-, cuando el éxito del argentino se hace realmente visible al nivel europeo. Labyrinthes, traducido ya al alemán en 1959, se reedita en 1962, y ese mismo año se vierte al inglés. El éxito de esta lectura ligeramente desviada de Borges por Caillois, centrada en la fascinación por el laberinto, se manifestaría de muchas maneras más, como la construcción del mayor laberinto del mundo por el editor de la legendaria revista de arte FMR, Franco Maria Ricci. Lo haría construir cerca de Parma y debe su idea original - dice- a su amigo Borges, con quien fundaría en 1974 una colección de literatura fantástica.

¿Qué se observa, ahora, girando la mirada hacia Alemania, donde Borges llegó poco después? La recepción de Borges parece haberse iniciado en 1956, con la publicación de La Forma de la espada traducido por Albert Theile (Die Spur des Schwertes), en una antología de cuentos latinoamericanos cuyo título hace competencia a la colección de Caillois: Unter dem Kreuz des Südens. Erzählungen aus Süd- und Mittelamerika ${ }^{16}$.

Pero un papel especial parece haber sido, por sus múltiples resonancias, el de Gustav René Hocke ${ }^{17}$, quien mencionó a Borges en su gran libro de 1957 sobre el manierismo de 1957: Die Welt als Labyrinth. Manier und Manie in der europäischen Kunst. El libro tuvo numerosas reediciones alemanas y tendría también, rápidamente, una traducción al castellano, en 1961. Hocke escribe acerca de Borges:

De Jorge Luis Borges aparecieron (en traducción francesa) Laberintos, narraciones que se desarrollan técnicamente de manera altamente laberíntica. En ellos se dice: "Yo soñaba hasta el agotamiento con un limpio, pequeño laberinto en cuyo centro estaba un ánfora, que mis manos casi tocaban, que mis ojos contemplaban, pero los senderos eran tan complicados como confusos, que se me hizo claro: moriría sin haber llegado jamás allí.” El

nues et des avenues, des rues et des rues. Ici, il n'y a pas de banlieue. Les quartiers excentriques, c'est déjà une banlieue perdue et noyée dans son propre désert. Tout cela semblait taillé à même le vide, car de chaque côté de ces voies trop larges et tellement longues, la lune écrasait des maisons imperceptibles."

16 En realidad, Albert Theile ya tradujo a Borges en Chile -así como a Mistral o Neruda-, en una revista que fundara allí en 1942 para oponerse al nazismo, los Deutsche Blätter - für ein europäisches Deutschland, gegen ein deutsches Europa. Sobre este asunto, véase Gerling (2007).

17 La importancia de Hocke para la recepción de Borges ya fue señalada por Gutiérrez Girardot (1964) (donde casi parafrasea el segundo texto de Hocke, de 1959, citado a continuación) y, luego, por Broyles-González (1981). 
'héroe' se encuentra ante un laberinto: "Yo no sé de cuántas piezas consta; mi desventura y mi angustia se aumentan sin cesar”. En la patria de Góngora, que murió en estado de perturbación mental, el motivo del laberinto tenía que seguir siendo alucinatorio ${ }^{18}$.

Habría mucho que decir sobre esta visión de un Borges compatriota de Góngora, que hace del argentino un heredero mucho más directo del manierismo andaluz, en una suerte de continuidad ideal.

Lo interesante es ver cómo Hocke traduce directamente del francés dos pasajes de El inmortal, que pudo consultar tanto en la edición de Labyrinthes de 1953 como en la edición separada del texto salida en la Nouvelle Revue Française, en septiembre del mismo año ${ }^{19}$. La traducción que Hocke da no es la que Karl-August Horst va a publicar poco después, en $1959^{20}$, y resulta curioso observar, además, cómo el traductor español de Hocke va a dar en 1961 otra versión del pasaje de Borges, notablemente alterado en comparación con la versión original ${ }^{21}$, pero que a su vez será citada unas cuantas veces por autores hispanohablantes deseosos de adornar sus

18 Traducción nuestra: "Von Jorge Luis Borgés [sic] erschienen (in französischer Übersetzung) 'Labyrinthes', Erzählungen, die sich technisch höchst labyrinthisch entwickeln. Darin heißt es: 'Unerträglich träumte ich von einem sauberen, kleinen Labyrinth, in dessen Mittelpunkt eine Amphora stand, die meine Hände fast berührten, die meine Augen sahen, aber die Umwege waren so kompliziert, so verwirrend, dass mir klar wurde: eher würde ich sterben als je dorthin gelangen'. Der 'Held' steht vor einem Labyrinth: 'Ich weiß nicht, um wie viel Zimmer es sich handelt; mein Unglück und meine Angst vervielfältigen sie dauernd.' In der Heimat des in geistiger Verstörtheit endenden Góngora musste das Labyrinth-Motiv so 'halluzinatorisch' bleiben" (Hocke 1957: 103).

19 Borges (1953: 425). Resulta interesante recordar que uno de los principales actores de la recepción de Borges en Alemania, el colombiano Rafael Gutiérrez Girardot - sobre quien se volverá enseguida-, citará años más tarde el pasaje de Hocke en un ensayo sobre Borges en Alemania, para resaltar que no logra identificar los pasajes aludidos por Hocke: "De Jorge Luis Borges aparecieron (en traducción francesa) Laberintos, narraciones que se desarrollan técnicamente de manera altamente laberíntica. En ellos se dice (siguen dos citas no identificables de Borges, porque son traducidas de la traducción francesa al alemán y Hocke no da la página). El 'héroe’ se encuentra ante un laberinto (sigue otra cita sobre el terror y la desdicha del héroe que se multiplican constantemente). En la patria de Góngora, que murió en estado de perturbación mental, el motivo del laberinto tenía que seguir siendo alucinatorio" (Gutiérrez Girardot 1998: 150).

20 Borges (1959): "Mich folterte unerträglich der Traum von einem eng zusammengezogenen gleißenden Labyrinth; in seiner Mitte war ein Krug; beinahe faßte ich ihn mit Händen, ich hatte ihn vor Augen, doch so verschlungen und vertrackt waren die Windungen, daß ich wußte, eher würde ich sterben, als an ihr herankommen".

21 Compárese esta versión española (por Rey Anciros) de la versión alemana (por Hocke) de la versión francesa (por Caillois): "Yo soñaba hasta el agotamiento con un limpio, pequeño laberinto en cuyo centro estaba un ánfora, que mis manos casi tocaban, que mis ojos contemplaban, pero los senderos eran tan complicados como confusos, que se me hizo claro: moriría sin haber llegado jamás allî" (Hocke 1961: 196), con el texto del mismo Borges: "Insoportable- 
textos con citas de Borges - de segunda mano, sin haberlo leído siempre-. Infinita movilidad de un texto que flota entre sus distintas versiones de un idioma a otro.

En 1959, Gustav René Hocke vuelve a mencionar a Borges, en la segunda parte de su libro, Manierismus in der Literatur. Sprach-Alchimie und esoterische Kombinationskunst:

Los cuentos del español [sic] Jorge Luis Borges (nacido 1899) llevan el título significativo Aleph, denominación hebrea de la letra A. Estas obras de prosa en todo punto alejandrinas son gongorescas, conscientemente elaboradas, desde el punto de vista estilístico, a partir de modelos del siglo XVII. Escribir laberintos supone, parece, crear relaciones inextricables, y abducir al lector a regiones "mágicas". Un laberinto simbólico se convierte, en los textos de Borges, en un "laberinto invisible del tiempo". Se piensa "en un laberinto de laberintos, en un sinuoso laberinto creciente que abarca el pasado y el porvenir y que [implica] de algún modo a los astros”22.

La palabra gongoresco, de claro origen italiano, conlleva connotaciones negativas en castellano ${ }^{23}$, pero no en inglés, mientras que en alemán (gongoresk) apenas se usa. Conviene, por lo tanto, no entenderla en un sentido despreciativo y menos cuando Hocke tiene precisamente como objetivo el desarrollo de una historia general del manierismo, del que el gongorismo no es sino la manifestación andaluza más genuina-. Resulta llamativa la insistencia de Hocke en designar a Borges como español cuando su descripción del laberinto borgeano se basa en una traducción que Herbert Kluger había publicado de El jardín de senderos que se bifurcan en 1958, en la revista Merkur - una de las revistas alemanas de referencia de la posguerra-: en la breve nota que acompaña su traducción, Kluger indicaba con absoluta claridad la argentinidad de Borges, al

mente soñé con un exiguo y nítido laberinto: en el centro había un cántaro; mis manos casi lo tocaban, mis ojos lo veían, pero tan intrincadas y perplejas eran las curvas que yo sabía que iba a morir antes de alcanzarlo" (Borges 1949: 11).

22 Traducción nuestra: "Die Erzählungen des Spaniers [sic] Jorge Luis Borges (geb. 1899) haben den bezeichnenden Titel 'Aleph', hebräische Bezeichnung für den Buchstaben 'A'. Diese in jedem Sinne alexandrinischen Prosastücke sind gongoresk, bewußt nach Vorbildern des 17. Jahrhunderts stilisiert. Labyrinthe schreiben heißt anscheinend unentwirrbare Beziehungen herstellen, den Leser in 'magische' Welten entführen. Ein symbolisches Labyrinth wird - bei BORGES - zu einem 'unsichtbaren Labyrinth der Zeit'. Gedacht wird an ein 'Labyrinth der Labyrinthe, an ein gekrümmtes, sich stetig ausdehnendes Labyrinth, das die Vergangenheit und Zukunft umschließt und die Sternenwelt in sich einbezieht'” (Hocke 1959: 22-23). 23 Véase por ejemplo la siguiente condena: "Entre nosotros, aunque por otro estilo, el culteranismo y el idioma Gongoresco, de cuyos resabios no están exentos los mejores ingenios del siglo décimo-sétimo, son otra prueba de las aberraciones a que conduce el extravío del gusto. Los retruécanos, los conceptillos, las trasposiciones violentas, las metáforas oscuras y alegorías incomprensibles, anduvieron por mucho tiempo en boga no solo en las producciones literarias, sino hasta en la conversación familiar" (Corradi 1843, t. I: 110 -modernizamos la grafía-). 
mismo tiempo que define su estilo como "curiosa alianza de elementos meditativos y al mismo tiempo modernos-manieristas"24.

Son aquellos, en todo caso, años en que el nombre de Borges empieza a circular en los ámbitos de lengua alemana. Rafael Gutiérrez Girardot recuerda que, el mismo año 1958, animó a Ernesto Grassi a que propusiera la edición de textos de Borges a la editorial Rowohlt -editorial en la que Hocke publicara tanto Die Welt als Labyrinth (1957) como Manierismus in der Literatur (1959)-. "Esto no se entiende ni interesa en Alemania", habrían sido las palabras del lector de la editorial ${ }^{25}$.

Y, sin embargo, se observa una rápida difusión alemana de Borges, siempre en relación con la figura del laberinto, que se ha convertido, desde Caillois, en una suerte de signatura de la escritura borgeana. Los Labyrinthe se publicaron en 1959 en Múnich, en la editorial Carl Hanser, con postfacio de Karl-August Horst ${ }^{26}$. El mismo Horst, el principal traductor de los Labyrinthe de Borges $^{27}$, llega a caracterizarle en términos que lo convierten - siempre a la zaga de Caillois y, ahora, de Hocke- en una especie de maestro por antonomasia, un “clásico” del laberinto:

Pero vemos también cómo, dentro del ámbito que designamos con los conceptos de "literatura” y de "filosofía," no hay nada que se pueda fijar a largo plazo. Cada obra que sale nuevamente publicada puede revelar una perspectiva que ha permanecido oculta por décadas, por siglos. Un poeta como el argentino Jorge Luis Borges, que se mueve con la seguridad de un funámbulo por los laberintos de la literatura europea, tiene con sus relatos la capacidad de modificar imprevisiblemente los horizontes y las constelaciones de esta literatura. Cada experiencia contemporánea puede transponer lo que ha sido hacia un estado de tensión distinto, y proyectarlo distintamente hacia el porvenir. Cuanto más rica en metamorfosis y, por consiguiente, cuanto más inagotable se manifiesta un texto litera-

24 En Borges (1958): "Jorge Luis Borges ist der führende, durch eine eigentümliche Verbindung von meditativen und modern-manieristischen Elemente gekennzeichnete Erzähler Argentiniens. Die von Herbert Kluger übersetzte Geschichte erschien 1948 in der Sammlung "Ficciones", die durch die französische Ausgabe (Gallimard, Paris, 1952) das Ansehen des Autors in Europa begründete” (traducción nuestra: “Jorge Luis Borges es el primer narrador de Argentina, caracterizado por una curiosa alianza de elementos meditativos y, al mismo tiempo, modernos-manieristas. La historia traducida por Herbert Kluger se había publicado en 1948 en la colección Ficciones, cuya versión francesa (Gallimard, París, 1952) funda el prestigio del autor en Europa").

25 Gutiérrez Girardot (1998: 147-148).

26 Borges (1959) (“Nachwort” de Horst en las páginas 291-297). Se reeditarían muchas veces, en 1962 y unas cuantas veces más, en la colección de bolsillo dtv, hasta las ediciones revisadas de Borges (1981 y 1992).

27 Sobre Horst como traductor, véase, a partir de un análisis de su traducción de El Aleph, Gerling (1999). 
rio, que modifica nuestra relación con él a la par que descansa en sí mismo, tanto más tendemos a designarlo como clásico ${ }^{28}$.

También el ya mencionado Rafael Gutiérrez Girardot, que luego toma sus distancias respecto a la línea descrita hasta aquí, caracteriza inicialmente a Borges como laberíntico, aunque insistiendo en la necesidad de su lectura filosófica. Escribe, en un artículo que le dedica en 1961 en la revista Merkur, y que se puede entender como una reseña de los Labyrinthe de 1959:

Al lector, la singular construcción de Borges se le antojará más filosófica que literaria. Recuerda la representación del mundo simbólica de un Baltasar Gracián y, de hecho, Borges reanuda con la tradición del barroco español, para la cual el cosmos no era, por decirlo con una palabra de Paul Fleming, sino "un laberinto poético de Dios”. El laberinto poético construido por Borges se ve distinto del a través del cual el jesuita Gracián pensó el mundo. Es un laberinto poético, cuya definición pasa por Nietzsche: es un "laberinto poético sin Dios", en el que el hombre no se pierde mientras esté ocioso y alegre, mientras se muestre lúcido. Esto no es un principio ateo, es un principio literario, que se podría resumir con palabras de Gottfried Benn, espiritualmente emparentado con Borges: "Dios es un mal principio estilístico" ${ }^{29}$.

Otros críticos seguirían por esta vía a lo largo de los años sucesivos: así un ensayo de 1965 de Marianne Kesting sobre El Hacedor ("Das hermetische Labyrinth. Zur

28 Traducción nuestra. "Wir sehen aber auch, wie innerhalb des Bereichs, den wir mit den Begriffen Literatur und Philosophie bezeichnen, nichts auf die Dauer festzulegen ist. Jedes neu erscheinende Werk kann eine Perspektive, die Jahrzehnte und Jahrhunderte lang verborgen war, sichtbar machen. Ein Dichter wie der Argentinier Jorge Luis Borges, der sich mit nachtwandlerischer Sicherheit in den Labyrinthen der europäischen Literatur bewegt, kann deren Horizonte und Konstellationen durch seine Erzählungen unabsehbar verändern. Jedes Erlebnis der Gegenwärtigkeit kann die Gewesenheit in einen anderen Spannungszustand versetzen und die Zukunft anders projizieren. Je wandlungsreicher und somit unerschöpflicher eine literarische Erscheinung ist, die in sich ruhend unser Verhältnis zu ihr transformiert, um so mehr sind wir geneigt, sie klassisch zu nennen" (Horst 1960: 528-529).

29 Traducción nuestra. "Die eigenwillige Konstruktion von Borges wird eher philosophisch als literarisch anmuten. Sie erinnert an die symbolistische Weltdarstellung eines Baltasar Gracián, und in der Tat: Borges knüpft an die Tradition des spanischen Barocks an, für den der Kosmos, um es mit einem Wort von Paul Fleming zu sagen, 'ein poetisches Labyrinth Gottes' war. Das poetische Labyrinth, das Borges gebaut hat, sieht anders aus als das, mit dem der Jesuit Gracián die Welt bedachte. Es ist ein poetisches Labyrinth, dessen Wege über Nietzsche führen - es ist ein 'poetisches Labyrinth ohne Gott', in dem der Mensch sich nicht verliert, solange er müßig und verspielt sein will, solange er seine Luzidität behält. Das ist kein atheistisches, sondern ein literarisches Prinzip, das sich in den Worten des mit Borges geistesverwandten Gottfried Benn zusammenfassen ließe: 'Gott ist ein schlechtes Stilprinzip”' (Gutiérrez Girardot 1961: 176-177). Véase también Gutiérrez Girardot (1962). Sobre la importancia de Benn en el descubrimiento de Borges por el joven Gutiérrez Girardot, véase Rivas (2009). 
Dichtung von Jorge Luis Borges”) ${ }^{30}$, $u$ otro de índole más general, de Erika Lorenz, alemana entonces instalada en Colombia, que presenta a Borges como "uno de los nuestros” ("El eco de la literatura hispanoamericana en la República Federal Alemana") ${ }^{31}$. Se podrían multiplicar los ejemplos de juicios de este tipo, más o menos desarrollados, más o menos sutiles en su simplificación de la obra borgeana, y que conformarían una larga retahíla de ensayos que, hasta ahora, reducen la obra de Borges a esta visión centrada en la fascinación del laberinto.

La mayoría de los críticos que ha contemplado esta historia de la primera recepción alemana de Borges, desde los artículos pioneros de Rafael Gutiérrez Girardot y Gustav Siebemann, han considerado que esta lectura de Borges iba mal encaminada y que, en cierto modo, Borges habría llegado a Alemania pese a ello, cuando creo, en cambio, que cabe considerar que llegó gracias a esta coyuntura muy específica.

Son como mínimo tres las razones que explican la relativa homogeneidad de las primeras caracterizaciones críticas de Borges en Alemania. La primera sería, obviamente, la influencia de la operación de traducción/interpretación llevada a cabo por Caillois en Francia, cuya importancia, como se ha visto, los críticos alemanes reconocen abiertamente, ya a finales de los años 1950. Se lee así, en un artículo de François Bondy publicado en una serie de artículos de Die Zeit (“Aus den Hauptstädten der Welt”), con fecha del 9 de enero de 1959, lo siguiente:

"Zeno", la obra maestra de Italo Svevo también ha sido reeditada, y Roger Caillois ha introducido al argentino Jorge Luis Borges, el autor de los curiosos Laberintos, uno de los mayores escritores vivos, que, dicho sea de paso, ningún editor alemán parece haber descubierto hasta la fecha ${ }^{32}$.

30 Kesting (1965, reed. 1965).

31 Escribe acerca de Borges, recordando la postura de Horst para apoyarse en ella: "El secreto de su éxito es muy complejo. En parte radica en el juguetón manejo de elementos formativos europeos. Artístico, amanerado, alejandrino ha sido llamado el libro por este motivo, pero no sin complacencia. Por otra parte, el cuento fantástico es universal, sobrepasa las fronteras históricas y geográficas, estableciéndose en todas partes y en todos los tiempos. Además, el cuento de Borges tiene unos rasgos estilísticos fundamentales que en Europa se señalan como características de la actual literatura de vanguardia: en lugar de la continuidad aparece la intensidad producida por impresiones simultáneas, a las que Horst llama fascinación; en lugar del sentido claramente definible aparece la casualidad intangible y llena de sentido; en lugar del estilo, la parodia del estilo; en lugar del realismo del 'así es', la ironía. [. . .] Estos rasgos y algunos otras dejan aparecer a Borges como uno de los nuestros” (Lorenz 1965: 41-42).

32 Traducción nuestra. “'Zeno', das Meisterwerk von Italo Svevo, ist gleichfalls neu aufgelegt worden, und Roger Caillois hat den Argentinier Jorge Luis Borges, den Verfasser der seltsamen 'Labyrinthe', eingeführt, einen der größten lebenden Schriftsteller - den übrigens gleichfalls 
Aunque Bondy no solo no conocía la traducción de Theile, y tampoco parece haber estado al tanto de que la publicación de Labyrinthe se preparaba para 1959, su caso muestra que la difusión del conocimiento de Borges en Francia y en Alemania también se ha de entender desde una perspectiva ideológicopolítica: Borges es, claramente, un autor de tendencia liberal. Es de notar, asimismo, que Bondy comparte con Drieu la Rochelle la voluntad de hacer de Borges un autor plenamente cosmopolita, un ciudadano del mundo: en esto, la serie "Aus den Hauptstädten der Welt" de Bondy hace eco a aquella de Drieu, "Villes du Monde" 33 .

$Y$ es que, en efecto, Bondy es un anticomunista notorio, miembro del Congreso por la libertad de la Cultura fundado en 1950, y editor de la revista Preuves, así como de la revista de lengua castellana Cuadernos del Congreso por la libertad de la Cultura, en la que publica en diciembre de 1955 la "Historia de los ecos de un nombre” de Borges. Unos años después, en 1961, publica en la revista alemana Der Monat una entrevista con Borges ${ }^{34}$. Esta percepción politizada de Borges debía resultar obvia, en aquellos años, ya que se le veía como un autor de la revista Sur, que había sido la primera revista, en el ámbito hispánico, en hablar del libro de David Rousset que denunciaba los crímenes de Stalin, L'Univers Concentrationnaire $(1946)^{35}$.

El que Borges haya sido percibido en la Alemania de los años 1960 como políticamente orientado, lo demuestra también, a contrario, su consideración muy negativa en la República Democrática Alemana de entonces. Así, Alfred Antkowiak (1925-1976) -traductor, entre otros, de Jorge Amado ${ }^{36}$, lector en la editorial Volk und Welt y, sobre todo, informador secreto de la Seguridad del Estado - juzga Labyrinthe "totalmente inútil" (gänzlich unbrauchbar), en un informe del 31 de mayo de 1961, y no se publicará a Borges en la República Democrática Alemana hasta los años $1980^{37}$.

noch kein deutscher Verleger entdeckt zu haben scheint” (Bondy 1959). Nótese, además, que la caracterización del punto de vista francés es una preocupación constante de Bondy, quien impartiría en el otoño 1966 unas clases sobre "Literatura moderna vista desde París" ("Moderne Literatur von Paris aus gesehen”), en la cátedra de Walter Höllerer (Technische Universität Berlin).

33 Sobre Borges cosmopolita, véase Berveiller (1973).

34 Bondy (1961).

35 Sobre ello, véase Klengel (2013).

36 Sobre la fortuna de J. Amado en la República Democrática Alemana, véase Locane, en este mismo volumen.

37 Véase Kirsten (2004: 116), quien cita el informe sobre Labyrinthe (SBB/HAA/Dep. 38, Aufbau/Gutachten Lateinamerika A-C). La misma editorial Volk und Welt acoge en cambio en los mismos años autores como C. Vallejo; véase Béhar (2019). 
La segunda razón sería el papel del concepto de manierismo y/o de barroco como categoría que permitió ubicar -y es destacable la insistencia de los artículos de la época en la importancia de la "ubicación" del autor, que se impone como un término entonces recurrente ${ }^{38}$-, ubicar, pues, a Borges en el momento de su "recepción". Y, en este sentido, no es solo la influencia, al fin y al cabo, extranjera, de Caillois, sino la alemana, y bastante fuerte, de Gustav René Hocke, que asoció a Borges con esta categoría meramente estética a partir de $1957^{39}$. Conviene en realidad distinguir entre las categorías de "barroco" como categoría estilística promovida en Alemania desde finales del s. XIX por Wölfflin, y de "manierismo", promovido especialmente por Hocke: calificar a Borges como "manierista", y no como "barroco", permite no amalgamarlo con aquellos autores que están llegando desde América bajo la etiqueta de "barrocos" o "neo-barrocos". Aunque esta diferencia no se aplica siempre, y también los hay que, unos años después, aplican a Borges el marbete de "barroco".

La tercera, quizás menos evidente hoy en día, para nosotros, está vinculada con la anterior: los principales actores de la historia de este primer momento de la recepción alemana de Borges forman una especie de comunidad intelectual, que comulga en un concepto más general de lo que es la literatura. Esta comunidad se define por lo que tanto Gustav René Hocke como Karl-August Horst fueron alumnos de Ernst Robert Curtius (1886-1956), como también lo fueron otras figuras de gran importancia en el contexto de la recepción de la literatura latinoamericana de aquellos años, como Walter Boehlich, quien, después de haber sido el asistente de Curtius en la universidad de Bonn, fuera a partir de 1957 el lector jefe en la casa editorial Suhrkamp, donde contribuyó de modo decisivo a la recepción de la literatura latinoamericana en Alemania ${ }^{40}$. Todos encontraban en Borges una perfecta ilustración moderna del estilo "gongoresco", también llamado estilo "alejandrino", o sea, "manierista": quizás la americanidad de Borges le confiriera además un toque de exotismo - pese al europeanismo reivindicado por el autor de Ficciones - que entrara en perfecta consonancia con las connotaciones estilísticas asianistas que el alejandrinismo implicaba desde la Antigüedad.

38 La cuestión de la "situación" surge tanto en el Cahier de L'Herne (1964) como en lo que se puede considerar el primer ensayo histórico crítico, y no simplemente ensayístico -como los hasta aquí mencionados-, sobre la recepción de Borges en Alemania, Siebemann (1966).

39 Klengel (2013) insiste, sin embargo, sobre la dimensión estetizante de la caracterización de Borges por Caillois, que le permitió alejarlo de interpretaciones demasiado políticas en la inmediata postguerra.

40 Sobre esta fascinante figura de las letras alemanas de la postguerra, véase Peitsch/Thein (2011). 
De hecho, el mismo concepto de manierismo encuentra su origen en la obra magna de Ernst Robert Curtius, Literatura europea y Edad Media Latina (Europäische Literatur und Lateinisches Mittelalter), publicada en 1948 y pronto traducida al castellano $^{41}$. En esta suerte de compendio del saber literario de la Edad Media, Curtius había ampliado el uso de la noción retórica de topos para, sacándolo de los manuales clásicos, aplicarla a la descripción de motivos recurrentes de la literatura europea, entre latín y lenguas romances. Como tal, Literatura europea y Edad Media Latina se convirtió en una biblia de los estudios romanísticos de los años 1950-1960, y contribuyó poderosamente a crear un nuevo concepto de la literatura en la inmediata postguerra ${ }^{42}$. Todavía Walter Boehlich, quien había ayudado a Curtius en la edición final de Literatura europea y Edad Media Latina, caracterizaría en 1961 a Curtius como el maestro de la definición de la literatura europea que es sinónimo, para él, de la Weltliteratur ${ }^{43}$-.

Y es que el concepto de literatura desarrollado por Curtius permite entender la rápida aceptación de Borges en Alemania. En efecto, en su libro publicado poco tiempo después de la gran catástrofe de la barbarie nacional-socialista, Curtius había sentado las bases para una comprensión profundamente europeísta y, tendencialmente, universalista, de la literatura, fundada en la idea de un patrimonio intelectual común, nutrido de una cultura latina que todas las naciones europeas tienen en común y que se manifiesta en la perennidad de las temáticas - los famosos topoi de la retórica, cuya moda se debe a Curtius-. Al mismo tiempo, este concepto de "lo literario" tiende hacia la idea de la autonomía de la literatura, desapegada de cualquier localismo y, sobre todo, de cualquier ideología. La mencionada sentencia de Horst que caracteriza a Borges como "funámbulo que se mueve por los laberintos de la literatura europea” parece en este sentido reveladora: para quienes consideraban que la literatura se definía esencialmente por su apego a la tradición latina y como ejercicio de escritura basado en las intertextualidades, Borges no podía sino aparecer como la perfecta encarnación del poeta doctus del s. XX. Lo aquí caracterizado no es lo hemos visto - sino una tendencia, que va a limitar cómo se leería a Borges. La línea más política de leer a Borges, con Bondy, no entra en conflicto con aquella propugnada por Hocke y Horst, más apolítica, fundada en la identificación del autor mediante determinadas categorías estéticas.

41 Curtius (1948 y 1955).

42 Sobre la influencia de Curtius, véase Richards (1983) y Berschin/Rothe (1989). Véanse también los análisis críticos de Michael Nehrlich respecto a la pertenencia de Curtius a la tradición liberal alemana, en Nerlich (1996 y 2000).

43 Boehlich (1961). 
Otros críticos tomarían sus distancias respecto a estas lecturas, subrayando que al propio Borges no le gustaba ser caracterizado como "barroco", o que su escritura también era deudora de otras influencias, incluso alemanas ${ }^{44}$, y esta sería la línea defendida por Rafael Gutiérrez Girardot (1928-2005), de quien se ha mencionado antes cómo intentó promover la traducción de Borges en Rowohlt en 1958, y que publicó, en el mismo año 1959 su ensayo en castellano, Jorge Luis Borges. Ensayo de interpretación, texto de 139 páginas editado por la editorial Ínsula en Madrid, y parcialmente censurado por, como dijo Gutiérrez Girardot, la “inquisición clerical” española. Y es que Gutiérrez Girardot conoce la obra de Borges por razones personales muy concretas: pocos años antes, en 1955-56, tuvo que reemplazarlo en un cargo rechazado por Borges en el Instituto Iberoamericano de la universidad sueca de Gotemburgo ${ }^{45}$. Esta es, sin embargo, otra historia, porque Gutiérrez Girardot, que partió más bien de una lectura nietzscheana y expresionista, no influyó en la primera caracterización de Borges como poeta del laberinto, poeta "manierista/barroco", caracterización criticada, matizada, relativizada, pero que ha dejado una huella indeleble, hasta hoy, en la recepción alemana de Borges ${ }^{46}$. En este momento, la evocación de Borges sirve para crear una continuidad en la evolución del manierismo, desde el siglo XVI hasta el siglo XX, al mismo tiempo que unifica el concepto de literatura, y los críticos que luego utilizan la noción de barroco, como Gutiérrez Girardot, lo hacen para distanciarse de la línea de lectura de Borges en clave manierista.

En este contexto, el epíteto "gongoresco", equivalente italianizante del castellano "gongorino", juega un papel de catalizador, remitiendo a un estilo alejandrino con el que Hocke identifica el de Borges. Resulta interesante ${ }^{47}$ subrayar la coincidencia en el tiempo con la categoría de "Dixie Gongorist" (algo como: gongorista de los estados sureños de EE. UU. o, en palabras de Carlos Fuentes, "un ilegible barroco del Mississippi" ${ }^{48}$ ) que Allen Tate utiliza por los mismos años para caracterizar, con intención denigrante, el estilo de Faulk-

44 Véase, por ejemplo, Dapía (1993).

45 Sobre la importancia del encuentro intelectual con Borges, véase Rivas (2009).

46 Entre muchos otros, Kapschutschenko (1981), Grüning (1985), Schmitz-Emans (1992 y 2011), Koneffke (1993), Angelino (2003), Geisenhanslüke (2011) y, recientemente, Galasso (2012).

47 Agradezco a Álvaro Santa Acuña el habérmelo señalado.

48 Fuentes (2000: 5). Fuentes desarrolla una reflexión en torno a esta designación, positiva en su opinion: "I think it was Allen Tate who disparagingly referred to Faulkner as a Dixie Gongorist, which I think is really the highest praise because it links Faulkner to this culture of the incomplete, of the voracious, of the intertextual which is the baroque. There is a culture of the Caribbean, I would say, that includes Faulkner, Carpentier, García Marquez, Derek Walcott, and Aimé Césaire, a trilingual culture in and around the whirlpool of the baroque which is the Caribbean, the Gulf of Mexico. Think of Jean Rhys’s Wide Sargasso Sea” (Fuentes 1984: 354). 
ner, a quien Borges defendiera también un tiempo ${ }^{49}$. Aunque no proceda aquí, cabría abrir un capítulo sobre los usos del gongorismo redescubierto por la generación del 1927 en las batallas literarias del s. XX y, más concretamente en los sucesivos intentos de definición de la literatura latinoamericana durante la postguerra $^{50}$. Su uso por Gustav René Hocke constituye en todo caso un hito importante de esta historia en Alemania.

Muchos aspectos de la primera recepción de Borges en Alemania quedan aún por estudiar, sobre todo desde el punto de vista de la historia editorial. Del análisis aquí propuesto se desprende, sin embargo, que su primera recepción se da en el marco muy contrastado de la Guerra Fría, en el que Borges aparece como un autor liberal, poco afín al socialismo. Pero ha sido también la propia estructura de la romanística alemana de entonces que, marcada por la figura señera de Ernst Robert Curtius y su concepto de manierismo, anhelaba desmarcarse de su aborrecido pasado político: en Borges puede encontrar a un autor del que puede apropiarse, asociándolo desde temprano con Góngora y con la historia de la modernidad literaria en lengua española. Mediante esta asociación, llevada a cabo por Gustav René Hocke, hace de Borges un autor “manierista”, llevándolo así a un canon clásico y universalista que sirve de horizonte para la descontextualización y recontextualización de la obra de Borges en la Alemania de los años 1950/60.

Tanto en Francia como en Alemania, en efecto, se observa una estetización de la obra de Borges ${ }^{51}$ que niega sus implicaciones políticas, estudiadas por A. Louis ${ }^{52}$, para mejor considerarlo como un autor que pertenece a una literatura universal, una Weltliteratur cuyo estudio Erich Auerbach reclama exactamente en los mismos años ${ }^{53}$. Sin embargo, mientras que la recepción de Borges en Francia, después del "momento Caillois", se encamina -especialmente a partir del magnífico Cahier de l'Herne que se publicara en su honor en 1964-, hacia un diálogo con las nuevas tendencias de la crítica literaria, con Blanchot, Foucault y Genette $^{54}$, la llegada de Borges a Alemania coincide con un momento de evolución complejo de la noción de barroco, a la que la de manierismo empieza a hacer competencia.

49 Véase Day (1980).

50 Sobre Góngora en las letras españolas del primer s. XX, véase Egido (2009), con reseña en Béhar (2010). Sobre la estela americana de Góngora, véase Guerrero (2012).

51 Véase Klengel (2013).

52 Véase Louis (2007).

53 Véase Auerbach (1952).

54 Roger (2011) y, sobre el aparente desinterés de Barthes por Borges, véase Cámpora (2013). 


\section{Bibliografía}

Adriaensen, Brigitte/Wijnterp, Lies (2013): “Borges leîdo a través de sus cubiertas. Un estudio de las ediciones y traducciones tempranas de Ficciones y El Aleph en el contexto de la recepción". En: Variaciones Borges 36, pp. 3-34.

Aizenberg, Edna (ed.) (1990): Borges and his Successors. Columbia: University of Missouri Press.

Anón. (1954): “Lettres étrangères”. En: La Table ronde 73, p. 163.

Angelino, Maria-Isabella (2003): "I labirinti: il dramma del percorso". En: Doctor virtualis. Rivista online di storia della filosofía medievale 2 ("Medioevo storico e medioevo fantastico in Jorge Luis Borges"), <https://doi.org/10.13130/2035-7362/44> (última visita: 29/02/2020).

Auerbach, Erich (1952): “Philologie der Weltliteratur”. En: Muschg, Walter/Staiger, Emil (eds.): Weltliteratur. Festgabe für Fritz Strich zum 70. Geburtstag. Berna: Francke, pp. 39-50 (trad. it. [2006]: Philologie der Weltliteratur. Filologia della literatura mondiale. Trad.: E. Salvaneschi. Castel Maggiore, Book Editore.).

Bachelard, Gaston (2011): El agua y los sueños. Ensayo sobre la imaginación de la materia. Trad. Ida Vitale. México: Fondo de Cultura Económica.

Batalla, Martín (2010): “Le concours amical: Néstor Ibarra, traductor de Borges". En: Variaciones Borges 30, pp. 83-109.

Béhar, Roland (2019): “'Zutiefst unzeitgemäß’: Hans Magnus Enzensberger, traducteur de la poésie de César Vallejo en langue allemande”. En: Béhar, Roland/Breysse-Chanet, Laurence/Salazar, Ina (eds.): La Parole impossible. Regards croisés autour de la traduction de César Vallejo, de Marina Tsvetaeva et de Paul Celan. París: Éditions Hermann, pp. 273-310.

Béhar, Roland (2010): “Recovecos y recurrencias del Barroco”. En: Revista de Libros 166, pp. 34-35.

Berschin, Walter/Rothe, Arnold (eds.) (1989): Ernst Robert Curtius. Werk, Wirkung, Zukunftsperspektiven. Heidelberg: Carl Winter.

Berveiller, Michel (1973): Le Cosmopolitisme de Jorge Luis Borges. París: Didier.

Boehlich, Walter (1961): “Die Einheit der europäischen Literatur”. En: Die Zeit 11, 10/03/1961.

Bondy, François (1961): "Gespräche mit zwei Autoren: Jorge Luis Borges und Witold Gombrowicz”. En: Der Monat 13/150, pp. 88-92.

Bondy, François (1959): "Paris - Metropole der Weltbürgerlichkeit. Schöpferische Aneignungskraft - Brecht, Dürrenmatt, Hochwälder auf der französischen Bühne”. En: Die Zeit, 9/01/1959.

Borges, Jorge Luis (1992): Das Aleph. Trad. Karl-Horst August/Gisbert Haefs. Fráncfort del Meno: Fischer Taschenbuch.

Borges, Jorge Luis (1981): Erzählungen 1949-1970. Trad. Karl-August Horst/Curt Meyer-Clason. Trad. revisada por Gisbert Haefs. Múnich: Carl Hanser.

Borges, Jorge Luis (1964): Der Zahir und andere Erzählungen. Trad. Eva Hesse/Karl August Horst. Nachwort Rafael Gutiérrez Girardot. Fráncfort del Meno: Insel Verlag.

Borges, Jorge Luis (1959): Labyrinthe: Erzählungen. Trad. Karl August Horst/Eva Hesse/ Wolfgang Luchting/Liselott Reger. Nachwort Karl August Horst. Múnich: Carl Hanser.

Borges, Jorge Luis (1958): “Der Garten der verschlungenen Pfade”. Trad. Herbert Kluger. En: Merkur 123, pp. 450-458. 
Borges, Jorge Luis (1953a): Labyrinthes. Trad. Roger Caillois. París: Gallimard.

Borges, Jorge Luis (1953b): “L'Immortel”. Trad. Roger Caillois. En: Nouvelle Nouvelle Revue

Française, 1/09/1953, pp. 422-439.

Borges, Jorge Luis (1951): Fictions. Trad. Néstor Ibarra/Paul Verdevoye. París: Gallimard.

Borges, Jorge Luis (1949): El Aleph. Buenos Aires: Losada.

Borges, Jorge Luis (1946): “Les ruines circulaires”, Trad. Paul Verdevoye. En: Confluences 11, pp. 131-136.

Borges, Jorge Luis (1944): “Assyriennes. La loterie de Babylone, La bibliothèque de Babel”. Trad. Néstor Ibarra. En: Lettres françaises 14, pp. 13-26.

Borges, Jorge Luis (1939): “L’Approche du caché”. Trad. Néstor Ibarra. En: Mesures 2, pp. 115-122.

Broyles-González, Yolanda (1981): The German Response to Latin American Literature and the Reception of Jorge Luis Borges and Pablo Neruda. Heidelberg: Carl Winter.

Cámpora, Magdalena (2013): “¿Y Borges? Sobre un desinterés de Barthes”. En: Variaciones Borges 36, pp. 53-63.

Corradi, Fernando (1843): Lecciones de elocuencia forense y parlamentaria, pronunciadas en el Ateneo. Madrid: Establecimiento tipográfico, t. I.

Curtius, Ernst Robert (1955): Literatura europea y Edad Media Latina. Trad. Margit Frenk/ Antonio Alatorre. México: Fondo de Cultura Económica.

Curtius, Ernst Robert (1948): Europäische Literatur und Lateinisches Mittelalter. Berna:

A. Francke.

Dapía, Silvia G. (1993): Die Rezeption der Sprachkritik Fritz Mauthners im Werk von Jorge Luis Borges. Colonia/Weimar/Viena: Böhlau.

Day, Douglas (1980): “Borges, Faulkner, and The Wild Palms". En: The Virginia Quarterly Review 56, pp. 109-118.

Drieu la Rochelle, Pierre (1934): “Villes du Monde. Solitude de Buenos-Ayres”. En:

L'Intransigeant, 19.809, 23 janvier 1934, p. 6. Reed. en: “Le poète et sa ville”. En: Sur les Écrivains. París: Gallimard, 1964, pp. 119-120. Y en: id. (2016): Chroniques des années 30. París: Max Chaleil.

Egido, Aurora (2009): El barroco de los modernos. Despuntes y pespuntes. Valladolid: Cátedra Miguel Delibes/Universidad de Valladolid.

Fuentes, Carlos (2000): “El destino de un libro". Diario de Colima, 4/02/2000, p. 5.

Fuentes, Carlos (1984): “Carlos Fuentes”. En: Plimpton, George (ed.): Writers at Work. The Paris Review Interviews. Sixth Series. Intr. Frank Kermode. Nueva York: Penguin Books.

Galasso, Norberto (2012): Jorge Luis Borges . . . Un intelectual en el laberinto semicolonial. Buenos Aires: Ediciones Colihue.

Gavagnin, Gabriella (2018): “Prime traduzioni di Borges in Italia. 1927-1937”. En: Rassegna iberistica 109, pp. 61-80.

Geisenhanslüke, Achim (2011): “Im Labyrinth des Nichtwissens. Jorge Luis Borges”. En: Dummheit und Witz. Poetologie des Nichtwissens. Paderborn: Fink, pp. 260-268.

Gerling, Vera Elisabeth (2015): “Jorge Luis Borges y su biblioteca de Babel alemana: (re-)lecturas de “El Aleph”". En: Iberoamericana XV/58, pp. 63-80.

Gerling, Vera Elisabeth (2007): “Die 'Spur des Schwertes' führt zur 'Narbe'. Das Werk Jorge Luis Borges' in deutscher Übersetzung als Bibliothek von Babel”. En: Revista de Lengua y Lingüística Alemanas 1, pp. 37-56. 
Gerling, Vera Elisabeth (1999): “Interpretar la obra de Borges: El Aleph en traducción alemana entre 1959 y 1992”. En: Romano-Sued, Susana (ed.): Borgesíada. Córdoba (Argentina): Topografía, pp. 93-105.

Grüning, Uwe (1985): “Der Gott des Labyrinths. Der Argentinier Jorge Luis Borges”. En: Grüning, Uwe: Moorrauch. Essays. Berlín: Union Verlag, pp. 111-117.

Guerrero, Gustavo (2012): "En sus dominios no se pone el sol. Neobarrocos y otros gongorinos en la poesía latinoamericana del siglo XX”. En: Roses Lozano, Joaquín (coord.): Góngora: la estrella inextinguible: magnitud estética y universo contemporáneo. Madrid: Sociedad Estatal de Acción Cultural, pp. 209-223.

Gutiérrez Girardot, Rafael (1998): Jorge Luis Borges: el gusto de ser modesto. 7 ensayos de crítica literaria. Santafé de Bogotá: Panamericana Editorial.

Gutiérrez Girardot, Rafael (1976): “Borges en Alemania”. En: Alazraki, Jaime (ed.): Jorge Luis Borges. Madrid: Taurus, pp. 288-292.

Gutiérrez Girardot, Rafael (1964): “Borges en Allemagne”. En: Cahier de l'Herne. París: Cino del Duca, pp. 205-208.

Gutiérrez Girardot, Rafael (1962): “Weltgeschichte als Niedertracht”. Merkur 16, 170, pp. 387-390.

Gutiérrez Girardot, Rafael (1961): “Jorge Luis Borges”. Merkur 15, 156, pp. 176-177.

Gutiérrez Girardot, Rafael (1959): Jorge Luis Borges, Ensayo de interpretación. Madrid: Ínsula. Hocke, Gustav René (1961): El manierismo en el arte europeo de 1520 a 1650 y en el actual. Trad. José Rey Anciros. Madrid: Ediciones Guadarrama.

Hocke, Gustav René (1959): Manierismus in der Literatur. Sprachalchimie und esoterische Kombinationskultur. Reinbeck: Rowohlt.

Hocke, Gustav René (1957): Die Welt als Labyrinth. Manier und Manie in der europäischen Kunst. Beiträge zur Ikonographie und Formgeschichte der europäischen Kunst von 1520 bis 1650 und der Gegenwart. Reinbeck: Rowohlt Taschenbuch Verlag.

Horst, Karl August (1960): “Versuch über den progressiven Charakter der europäischen Literatur”. En: Merkur 14 (148), pp. 528-529.

Kapschutschenko, Ludmila (1981): El laberinto en la narrativa hispanoamericana contemporánea. Londres: Tamesis.

Kesting, Marianne (1965): "Das hermetische Labyrinth. Zur Dichtung von Jorge Luis Borges". En: Neue Deutsche Hefte 103, pp. 107-124, reed. en Kesting, Marianne (1965): Vermessung des Labyrinths. Studien zur modernen Ästhetik. Fráncfort del Meno: S. Fischer.

Kirsten, Jens (2004): Lateinamerikanische Literatur in der DDR: Publikations- und Wirkungsgeschichte. Berlín: Ch. Links Verlag.

Klengel, Susanne (2013): “'El universo (que otros Ilaman la Biblioteca)' y L'univers concentrationnaire: la recepción de Borges en la Francia de la segunda posguerra”. En: Variaciones Borges 36, pp. 35-51.

Koneffke, Jan (1993): Im Labyrinth der Fiktionen - Zu Borges. Berlín: Aufbau-Verlag.

Larbaud, Valéry (2011) [1925]: "Sobre Borges". Trad. John Jairo Gómez Montoya. En: Mutatis mutandis 4/1, pp. 89-92.

Larbaud, Valéry (1976) [1925]: "Sobre Borges”. Trad. Jaime Alazraki. En: Alazraki, Jaime (ed.): Jorge Luis Borges, El escritor y la crîtica. Madrid: Taurus, pp. 27-28.

Larbaud, Valéry (1925): "Sur Borges”. En: La Revue Européenne 3, VI-34.

Locane, Jorge (en prensa): “Literatura comunista mundial. Jorge Amado en la República Democrática Alemana y China”. En: este mismo volumen. 
Lorenz, Erika (1965): “El eco de la literatura hispano-americana en la República Federal Alemana”. En: Ideas Valores 7/25-26, pp. 27-43.

Louis, Annick (2020a): "El Aleph de Roger Caillois en Gallimard o de cómo salir del laberinto". En: Guerrero, Gustavo/Locane, Jorge J./Loy, Benjamin/Müller, Gesine (eds.): Literatura latinoamericana mundial. Dispositivos y disidencias. Berlín/Boston: De Gruyter, pp. 125-146.

Louis, Annick (2020b): "La traduction dans la revue Lettres françaises (1941-1947) de Roger Caillois”. En: Béhar, Roland/Camenen, Gersende (eds.): Scènes de la traduction France/ Argentine. París: Éditions rue d'Ulm.

Louis, Annick (2013): “Étoiles d'un ciel étranger : Roger Caillois et l’Amérique Latine”. En: Littérature 2/170, pp. 71-81.

Louis, Annick (2007): “Borges Mode d'emploi français”. En: Louis, Annick: Borges face au fascisme II. Les fictions du contemporain. Montreuil: Aux lieux d'être, pp. 307-323.

Louis, Annick (2002): “Caillois-Borges, ou qu'est-ce qui s’est passé ?”. En: Courtois, JeanPatrice/Krzywkowski, Isabelle (eds.): Diagonales sur Roger Caillois, Syntaxe du monde, paradoxe de la poésie. París: L'Improviste, pp. 81-101.

Molloy, Sylvia (1972): La diffusion de la littérature en France au XXe siècle. París: PUF.

Nerlich, Michael (2000): “Une nation méconnue: L'image de la France dans l’Université Allemande". En: Parfait, Nicole (ed.): Une entente de raison. La chute du Mur de Berlin et les relations franco-allemandes. París: Desjonquères, pp. 107-128.

Nerlich, Michael (1996): “Romanistik: Von der wissenschaftlichen Kriegsmaschine gegen Frankreich zur komparatistischen Konsolidierung der Frankreichforschung”. En: Cahiers d'Histoire des Littératures Romanes. Romanistische Zeitschrift für Literaturgeschichte 20, pp. 396-434.

Peitsch, Helmut/ Thein, Helen (eds.) (2011): Boehlich: Kritiker. Berlín: Akademie Verlag.

Pérez Blázquez, David (2018): "La recepción de las letras borgianas en Alemania: el caso de 'La biblioteca de Babel’”. En: Mutatis Mutandis. Revista Latinoamericana de Traducción, 11, 2 ("Latinoamérica traducida: caminos y destinos de la literatura latinoamericana contemporánea entre las lenguas”), pp. 418-437.

Proust, Marcel (1970-1993): Correspondance. Ed. Philip Kolb. París: Plon, 21 vol.

Richards, Earl Jeffrey (1983): Modernism, Medievalism and Humanism. A Research Bibliography on the Reception of the Works of Ernst Robert Curtius. Tubinga: Max Niemeyer.

Rivas, Carlos (2009): “El joven Rafael Gutiérrez Girardot. Apuntes para una biografía intelectual: el descubrimiento de Jorge Luis Borges y su estimulante influencia”. En: Co-herencia 6/11, pp. 53-65.

Roger, Julien (2011): “Genette, el otro de Borges”. En: Cámpora, Magdalena/González, Javier (eds.): Borges-Francia. Buenos Aires: Facultad de Filosofía y Letras de la Universidad Católica Argentina, pp. 109-118.

Schmitz-Emans, Monika (2011): “Labyrinthische Bücher - Jorge Luis Borges und die bildende Kunst. Festvortrag im Rahmen der Akademischen Feier zum 65. Geburtstag von Manfred Schmeling”. En: Universitätsreden 83. Sarrebruck: Universitätsverlag des Saarlandes, pp. 17-36.

Schmitz-Emans, Monika (1992): "Lesen und Schreiben nach Babel. Über das Modell der labyrinthischen Bibliothek bei Jorge Luis Borges und Umberto Eco”. En: Arcadia 27/1-2, pp. 106-124. 
Siebenmann, Gustav (1996): "Sind die Deutschen die letzten Entdecker Amerikas? Zur Rezeption der lateinamerikanischen Literaturen”. En: Kohut, Karl et al. (ed.): Deutsche in Lateinamerika - Lateinamerika in Deutschland. Fráncfort del Meno: Vervuert, pp. 297-314.

Siebenmann, Gustav (1992): "Ein deutsches Requiem für Borges. Feststellungen und Materialien zu seiner Rezeption”. En: Iberoromania 36, pp. 52-72.

Siebenmann, Gustav (1966): “Jorge Luis Borges - ein neuer Typ des lateinamerikanischen Schriftstellers”. En: Germanisch-romanische Monatsschrift 16, pp. 297-314.

Topuzian, Marcelo (2014): "La literatura mundial como provocación de los estudios literarios". En: Chuy. Revista de estudios literarios latinoamericanos 1, <http://iflh.institutos.filo. uba.ar/sites/iflh.institutos.filo.uba.ar/files/RevistaChuy_1_1_Topuzian_literatura.pdf> (última visita: 29/ 02/2020).

Tuzet, Hélène (1984): “Rencontre de Bachelard avec Gabriele d’Annunzio”. En: Revue de Littérature Comparée 2, pp. 215-233.

Zangwill, Israel (1924): “'Lachrymae rerum’ à Mantoue (avec une pierre dans le jardin de d’Annunzio . . . )”. En: Zangwill, Israël: Fantaisies italiennes. Trad. $M^{\text {me }}$ Marcel Girette. París: Les Éditions C. Crès, pp. 1-30.

Zangwill, Israel (1910): “Lachrymæ rerum at Mantua: With a Denunciation of d'Annunzio". En: Zangwill, Israël: Italian Fantasies. Londres: William Heinemann, pp. 214-226. 
